\title{
STRUCTURE OF PSEUDO-SEMISIMPLE RINGS
}

\author{
SAAD MOHAMED and BRUNO J. MÜLLER
}

(Received 2 May 1989; revised 10 October 1989)

Communicated by B. J. Gardner

\begin{abstract}
A ring $R$ is called right pseudo-semisimple if every right ideal not isomorphic to $R$ is semisimple. Rings of this type in which the right socle $S$ splits off additively were characterized; such a ring has $S^{2}=0$. The existence of right pseudo-semisimple rings with zero right singular ideal $Z$ remained open, except for the trivial examples of semisimple rings and principal right ideal domains. In this work we give a complete characterization of right pseudo-semisimple rings with $S^{2}=0$. We also give examples of non-trivial right pseudo-semisimple rings with $Z=0$; in fact it is shown that such rings exist as subrings in every infinite-dimensional full linear ring. $A$ structure theorem for non-singular right pseudo-semisimple rings, with homogeneous maximal socle, is given. The general case is still open.
\end{abstract}

1980 Mathematics subject classification (Amer. Math. Soc.) (1985 Revision): primary 16 A 48; secondary 16 A 42,16 A 52.

Throughout this paper, $S, Z$ and $J$ will stand for the right socle, the right singular ideal and the Jacobson radical of a ring $R$. A local ring $R$ will mean one in which $J \neq 0$ and $R / J$ is a division ring. For a subset $X$ of $R, X^{0}$ and ${ }^{0} X$ will denote the right and left annihilators in $R$. It is true in general that $S \leq{ }^{0} J$, and if $R / J$ is semisimple (in particular if $R$ is local), then $S={ }^{0} J$. We also note that $Z$ and $J$ contain no non-zero idempotents of $R$; hence a regular ring $R$ has $Z=J=0$.

The split extension $R \rtimes M$ of a ring $R$ by an $(R-R)$-bimodule $M$, is the ring of all ordered pairs $(r, m), r \in R$ and $m \in M$; with addition defined componentwise and multiplication defined by $(r, m) \cdot\left(r^{\prime}, m^{\prime}\right)=$ $\left(r r^{\prime}, r m^{\prime}+m r^{\prime}\right)$.

This research was supported in part by the NSERC of Canada, grants A4033 and A8778.

(C) 1991 Australian Mathematical Society 0263-6115/91 \$A2.00+0.00 
The set of positive integers will be denoted by $\mathbb{N}$.

\section{General results}

Lemma 1.1. Let $R$ be a right pseudo-semisimple ring. If $R=A \oplus B$ for right ideals $A$ and $B$, then $A$ or $B$ is semisimple.

Proof. Assume that $A$ is not semisimple; then $A \cong R$. Hence $A=$ $A_{1} \oplus B_{1}$ with $A_{1} \cong A \cong R$ and $B_{1} \cong B$. Iterating this process, we obtain $R=A_{n} \oplus B_{n} \oplus \cdots \oplus B_{1}$ for every $n \in \mathbb{N}$. Hence $R$ contains the right ideal $\bigoplus_{i \in \mathbb{N}} B_{i}$ with $B_{i} \cong B$. This right ideal is not finitely generated, and therefore not isomorphic to $R$. Thus it is semisimple, and hence $B$ is semisimple.

Corollary 1.2. Let $R$ be a right pseudo-semisimple ring which is not semisimple.

(1) If $R=A \oplus B$ for non-zero right ideals $A$ and $B$, then exactly one of them is semisimple and the other one is isomorphic to $R$. In particular neither $A$ nor $B$ is an ideal.

(2) If $e$ is a non-trivial idempotent of $R$, then $e R(1-e) \neq 0$.

LEMMA 1.3. If $R$ is right pseudo-semisimple, then $R / S$ is a principal right ideal domain.

Proof. It is obvious that $R / S$ is a principal right ideal ring. Consider $a, b \in R$ with $a \notin S$ and $a b \in S$. Then $a R \cong R$, and hence $R=a^{0} \oplus C$ where $C \cong a R \cong R$. Thus $a^{0} \leq S$ by Lemma 1.1. Write $b=x+y$ with $x \in a^{0}$ and $y \in C$. Since $S \geq a b R=a y R \cong y R$, we have $y \in S$. Therefore $b=x+y \in S$.

It follows by Lemma 1.3 that a ring $R$ with zero right socle is right pseudosemisimple if and only if it is a principal right ideal domain. We call a right pseudo-semisimple ring $R$ non-trivial if $0 \neq S \neq R$.

LEMMA 1.4. Let $R$ be a non-trivial right pseudo-semisimple ring. The following hold in $R$ :

(1) $S$ is the smallest essential right ideal;

(2) ${ }^{0} S=Z \leq S \cap J$;

(3) $S=Z \oplus I$ where $Z$ and I consist of homogeneous components (hence $I$ is also an ideal);

(4) $S={ }^{0} x$ for every $0 \neq x \in J$, in particular if $J \neq 0$, then $S={ }^{0} J$; 
(5) if $a \notin S$, then $a R \cap S=a S$ and $S=a S \oplus K$ where $K$ is isomorphic to a direct summand of $R$ (hence finitely generated);

(6) $S Z=0$ and $Z$ is torsion-free divisible as a left $R / S$-module.

Proof. (1) Consider a non-zero right ideal $A$ of $R$. Then either $A \leq S$ or $A \cong R$, and hence $A$ contains a copy of $S$. In either case $A \cap S \neq 0$.

(2) $Z={ }^{0} S$ follows by (1). This also proves that $Z \not Z R$ and hence $Z \leq S$. Then $Z^{2} \leq Z S=0$, and consequently $Z \leq J$.

(3) If $X$ and $Y$ are minimal right ideals with $X \leq Z$ and $Y \not Z$, then $X S=0$ and $Y S \neq 0$. Thus $X \not \equiv Y$.

(4) That $S \leq{ }^{0} J \leq{ }^{0} x$ is obvious. Consider an element $a \in R$ such that $a \notin S$. Then $a^{0} \leq S$ and is a direct summand of $R$ (since $a R \cong R$ ). Therefore $a^{0} \cap J=0$ and hence $a x \neq 0$. This proves that ${ }^{0} x \leq S$, and consequently $S={ }^{0} J={ }^{0} x$.

(5) That $a S=a R \cap S$ follows by Lemma 1.3. Let $S=a S \oplus K$. Then $R \cong a R+S=a R \oplus K$.

(6) The result is trivial in the case $Z=0$. Assume that $Z \neq 0$. Since $Z \leq J, S={ }^{0} Z$ by (4). Hence $Z$ is a torsion-free $R / S$-module.

Next we prove that $Z \leq A$ for every right ideal $A$ not contained in $S$. Write $Z=(A \cap Z) \oplus B$. Then $A \oplus B \cong R$. Since $B \leq Z, B^{2}=0$ by (2), and hence $B=0$. Thus $Z=A \cap Z$ and $Z \leq A$.

Now consider an element $a \in R$ such that $a \notin S$. Since $a R \cong R, a R=$ $b R$ with $b^{0}=0$. If $b r \in Z$, then $b r S=0$, and hence $r S=0$ and $r \in Z$ by (2). Therefore $b R \cap Z=b Z$. Now

$$
a Z=a R Z=b R Z=b Z=b R \cap Z=Z .
$$

Hence $Z$ is divisible as a left $R / S$-module.

LEMMA 1.5. Let $R$ be a non-trivial right pseudo-semisimple ring.

(1) $Z=S$ if and only if $S^{2}=0$ and $S \leq J \leq S^{0}$.

(2) $Z \neq S$ if and only if $S$ contains a countable set of non-zero orthogonal idempotents and $S^{0}=J<S$.

Proof. (1) is obvious.

(2) Assume that $Z \neq S$. Then $S^{2} \neq 0$, and hence $S$ contains a non-zero idempotent. By an argument similar to that given in Lemma 1.1, we conclude that $S$ contains a countable set of non-zero orthogonal idempotents $\left\{e_{n}\right\}$.

Write $S=(J \cap S) \oplus X$. It is clear that the projections of the $e_{n}$ into $X$ are still non-zero orthogonal idempotents, and hence $X$ is not finitely generated. It then follows by Lemma 1.4(5) that $J \not \neq R$. Thus $J \leq S$, and since $S^{2} \neq 0, J<S$. 
Let $B=S^{0}$. Then $J \leq B$ and so $J \leq B \cap S$. Moreover $(B \cap S)^{2}=0$, and hence $B \cap S \leq J$. Thus $B \cap S=J$ and $S=(B \cap S) \oplus X$. Again by Lemma 1.4(5) we get $B \not \nexists R$ and hence $B \leq S$. Therefore $B=B \cap S=J$.

The converse is obvious.

COROLLARY 1.6. If $R$ is a right pseudo-semisimple ring, then the right socle of $R$ contains the left socle.

Proof. The result is obvious in the trivial cases. So assume that $R$ is non-trivial and consider the two cases of Lemma 1.5.

If $Z=S$, study a minimal left ideal $A$. Assume that $A^{2} \neq 0$. Then $A=\operatorname{Re}$ for some idempotent $e$ such that $0 \neq e \neq 1$. However, by Lemma 1.1 , either $e \in S$ or $1-e \in S$, in contradiction to $S^{2}=0$. Hence $A^{2}=0$, and $A \leq S$ by Lemma 1.3 .

If $Z \neq S$, then $S^{0}<S$ by Lemma 1.5 . It follows readily that $S$ is essential as a left ideal, and therefore contains the left socle.

The following generalization of our theorem in [3] characterizes right pseudo-semisimple rings with socle square zero.

THeOREM 1.7. Let $R$ be a ring with $S^{2}=0$. Then $R$ is right pseudosemisimple if and only if $R / S$ is a principal right ideal domain and $S$ is torsion-free divisible as a left $R / S$-module.

Proof. The 'only if part follows from Lemma 1.3 and Lemma 1.4 ((2) and (6)).

Conversely, assume that $R$ satisfies the conditions. Consider a right ideal $A$ of $R$ which is not contained in $S$, and select $x \in A-S$. Since ${ }_{R / S} S$ is divisible, $S=x S \leq A$. Now $R / S$ is a principal right ideal domain implies $A=a R$ with $a^{0} \leq S$. But then $a^{0}=0$ as ${ }_{R / S} S$ is torsion-free. Hence $A=a R \cong R$.

According to Lemma 1.5, a non-trivial right pseudo-semisimple ring with $S^{2}=0$ satisfies $0 \neq S \leq J \leq S^{0}$. We list examples of the four possible cases.

EXAMPLES 1.8. (1) $S=J=S^{0}$ : any local ring $R$ with $J^{2}=0$.

(2) $S=J<S^{0}: R=F[X] \rtimes F(X)$, the split extension of the polynomial ring over a field $F$ by the rational function field, made into an $F[X]-$ bimodule via the natural multiplication on the left and multiplication by the constant coefficient on the right; compare [3].

(3) $S<J=S^{0}$ : the localization of (2) at $\langle X\rangle$.

(4) $S<J<S^{0}$ : the localization of (2) at $\langle X\rangle \cap\langle X+1\rangle$. 
THEOREM 1.9. Let $R$ be a ring with $S \neq 0$ and $S^{2}=0$. Then $R$ is right and left pseudo-semisimple if and only if $R$ is a local ring with radical square zero.

Proof. The 'if part is obvious. Conversely, assume that $R$ is right and left pseudo-semisimple. Then the left-right analogue of Corollary 1.6 implies that $S$ is the left socle of $R$. Consider a minimal left ideal $A$. Since $A^{2}=0, A=R x$ with $x \in J$, and $A \cong R /{ }^{0} x$. Since $S={ }^{0} x$ by Lemma 1.4(4), $S$ is a maximal left ideal. Then $S=J$ as $S \leq J$ and the result follows.

Proposition 1.10. Let $R$ be a non-trivial right pseudo-semisimple ring. Then $R / Z$ is right pseudo-semisimple with $Z(R / Z)=0$. Moreover $R / Z$ is semisimple if and only if $R$ is a local ring with radical square zero.

Proof. Let $T / Z$ be the right socle of $R / Z$. Then it is obvious that $S \leq T$. Moreover $T / S$ is contained in the right socle of $R / S$. Since $R / S$ is a domain by Lemma $1.3, T=S$ or $T=R$.

(i) Consider the case $T=S$. Let $A / Z$ be a right ideal of $R / Z$ such that $A / Z \Varangle S / Z$. Then $A \Varangle S$ and hence $A=a R$ for some $a \in R$ with $a^{0}=0$. Since $Z=a Z$ by Lemma $1.4(6)$, we obtain

$$
R / Z \cong a R / a Z=A / Z \text {. }
$$

Thus $R / Z$ is right pseudo-semisimple.

Next we prove that $Z(R / Z)=0$. If $Z=S$, then $R / Z$ is a domain by Lemma 1.3 , and the result holds trivially. So, assume that $Z \neq S$. Then by Lemma 1.4(3), $S=Z \oplus I$ for a non-zero ideal $I$ of $R$. Consider $x+Z \in Z(R / Z)$. Then $x S \leq Z$, and hence $x I \leq Z \cap I=0$. Consequently $x R \not \equiv R$, and therefore $x \in S$. Then $x Z=0$ by Lemma 1.4(6), and $x S=x(Z \oplus I)=0$. Thus $x \in Z$.

(ii) Now, assume that $T=R$. Then $R / Z$ is semisimple, and clearly $Z(R / Z)=0$. We claim that $Z$ is a maximal ideal. Let $u$ be a central idempotent in $R / Z$. Since $Z$ is a nil ideal by Lemma 1.4(2), we may assume that $u=e+Z$ for some idempotent $e \in R$. According to Lemma $1.1, e \in S$ or $(1-e) \in S$; we may assume that $e \in S$. Then $Z \cap e R=0$. Since $e R(1-e) \leq Z, e R(1-e)=0$. It then follows by Corollary 1.2(2) that $e=0$ or $e=1$. Thus $R / Z$ has no non-trivial central idempotents, and is therefore simple artinian. This proves our claim. Since $Z \leq S \cap J$ by Lemma $1.4(2)$, we obtain $S=J=Z$. It then follows by Lemma 1.3 that 
$R / J$ is a division ring. Hence $R$ is a local ring with $J^{2}=0$. The rest is obvious.

\section{Maximal socle}

We turn to the second type in Lemma 1.5. Here we do not know of an effective criterion for pseudo-simplicity. However, right pseudo-semisimple rings $R$ of this type are characterized in the special case where $S$ is a maximal right ideal. In view of Lemma 1.3, this additional assumption is automatically satisfied if $R$ is regular.

We start by listing some properties of rings $R$ with maximal socle. Note that such rings are local if and only if $S^{2}=0$. The proofs are straightforward, and hence are omitted.

LEMMA 2.1. Let $R$ be a non-local ring with maximal socle (that is, $R / S$ is a division ring and $\left.S^{2} \neq 0\right)$. Then $R$ has the following properties:

(1) $S$ is the only proper essential right ideal;

(2) every right ideal is semisimple or a direct summand;

(3) if $R=A \oplus B$ for right ideals $A$ and $B$, then precisely one of them is semisimple,

(4) ${ }^{0} S=Z \leq J<S$, and $J^{2}=0$;

(5) $J \leq A$ for every right ideal $A$ not contained in $S$;

(6) $R$ is regular if and only if $J=0$ if and only if $R$ is semiprime.

Consider an idempotent $g$ in the socle of an arbitrary ring $R$. It is well known that $(1-g) R \cong R$ holds if and only if $R \cong R \oplus g R$ if and only if there exist $t, t^{*} \in R$ such that $t^{*} t=1$ and $t t^{*}=1-g$ (hence $R(1-g) \cong R$ also holds). We call $t$ a shift for $g$.

Now assume that for every isomorphism type of indecomposable idempotents $f$ in $S$, there is a representative $f^{\prime}$ for which there exists a shift. Then $R \oplus f R \cong R \oplus f^{\prime} R \cong R$. It follows that $R$ has a shift for every idempotent $e \in S$. Indeed, $e R=\bigoplus_{i=1}^{n} e_{i} R$ with $e_{i}$ indecomposable, and hence

$$
R \oplus e R=R \oplus e_{1} R \oplus \cdots \oplus e_{n} R \cong R \text {. }
$$

Such a ring $R$ is said to have enough shifts.

THeOREM 2.2. Let $R$ be a non-local ring with maximal socle. Then $R$ is right pseudo-semisimple if and only if $R$ has enough shifts.

Proof. From (1) and (2) of Lemma 2.1, the proof is obvious. 
COROLlaRy 2.3. Let $R$ be a ring with maximal socle. The following are equivalent:

(1) $R$ is right and left pseudo-semisimple and regular;

(2) $R$ is right pseudo-semisimple and $J=0$;

(3) $R$ is semiprime and has enough shifts.

Proof. That (1) implies (2) and that (2) implies (3) are obvious.

Assume (3). Since $R$ is semiprime, $S^{2} \neq 0$ and therefore $R$ is non-local. Then (3) implies (1) follows from Lemma 2.1(6) and Theorem 2.2.

The next proposition effectively reduces the study of pseudo-simplicity for rings with maximal socle to the non-singular case.

Proposition 2.4. Let $R$ be a non-local ring with maximal socle. Then $R / Z$ is right pseudo-semisimple if and only if $R$ is right pseudo-semisimple or $R=A \oplus B$, where $A$ is a local ring with radical square zero and $B$ is semisimple.

Proof. It is clear that $R / Z$ is semisimple for any ring $R=A \oplus B$ as described above; the 'if' part then follows from Proposition 1.10. Conversely, assume that $\bar{R}=R / Z$ is right pseudo-semisimple. The right socle of $\bar{R}$ can either be $\bar{S}$ or $\bar{R}$.

In the first case, for any right ideal $C \not S$ we have $\bar{C}=\overline{c R}$ with $c^{0} \leq Z$. Since $Z \leq C$ by Lemma $2.1((4)$ and (5)), we obtain $C=c R$. Also $C$ is a direct summand of $R$, and hence is projective. Thus $c^{0}$ is a direct summand of $R$, and consequently $c^{0}=0$. Therefore $C \cong R$, and $R$ is right pseudo-semisimple.

In the second case, we have $Z=J$ and $J^{2}=0$. Since $\bar{R}$ is semisimple, $\bar{R}=\bigoplus_{i=1}^{n} T_{i}$, where each $T_{i}$ is a simple artinian ring. Then $1=\sum_{i=1}^{n} e_{i}$ where the $e_{i}$ are orthogonal idempotents of $R, \bar{e}_{i}$ is central in $\bar{R}$ and $\bar{e}_{i} \bar{R}=T_{i}$. Since for $i \neq j, e_{i} e_{j}=0$ and $R / S$ is a domain, all the $e_{i}$, except possibly one, are in $S$. We denote the exceptional one by $e$. By Lemma 2.1(3), $(1-e) R \leq S$ and therefore $(1-e) R e \leq Z \cap(1-e) R=0$. Also

$$
e R(1-e) \leq Z={ }^{0} S \leq{ }^{0}(1-e)=\operatorname{Re},
$$

and hence $e R(1-e)=0$. Thus $e$ is a central idempotent in $R$. Let $A=e R e$ and $B=(1-e) R(1-e)$. Then $B$ is semisimple. As $A / J(A)=\overline{e R}$ is simple artinian, $J(A)$ is a maximal ideal in $A$. However $J(A)=A \cap J \leq A \cap S$; and so $J(A)=A \cap S$. Now $A / J(A)=A /(A \cap S) \cong A+S / S=R / S$, a division ring. It is obvious that $J(A) \neq 0$ and $J(A)^{2}=0$. Hence $A$ is a local ring with radical-square zero. 
COROLlaRY 2.5. Let $R$ be a non-local ring with homogeneous maximal socle. Then $R$ is right pseudo-semisimple if and only if $R / Z$ is.

We end this section by showing that any non-trivial right pseudo-semisimple ring $R$ with $Z=0$ can be embedded in one with maximal socle.

Proposition 2.6. Let $R$ be a non-trivial right pseudo-semisimple ring with $Z=0$. Then $R$ is isomorphic to a subring of a right pseudo-semisimple ring $R_{*}$ with $Z\left(R_{*}\right)=0$ and $S\left(R_{*}\right)$ maximal.

Proof. Let $\Sigma=\left\{c \in R: c^{0}=0\right.$ and $\left.c S=S\right\}$. Clearly $\Sigma$ is multiplicatively closed and $1 \in \Sigma$. If $x c=0$ for $x \in R$ and $c \in \Sigma$, then $x S=x c S=0$; hence $x \in Z=0$. Thus $\Sigma$ consists of regular elements. Now we prove that $\Sigma$ is a right Öre set. Let $c \in \Sigma$ and $r \in R$. If $r \in S$, then $r \in c S$; consequently $r 1=c r^{\prime}$ with $r^{\prime} \in R$. Assume that $r \notin S$, and let $B=\{b \in R: r b \in c R\}$. It is clear that $S \leq B$. If $S=B$, then $c R \cap r R=r B \leq S$. This implies $\overline{c R} \cap \overline{r R}=0$ in $\bar{R}$, in contradiction of the fact that $\bar{R}$ is a principal right ideal domain. Thus $S<B$, and therefore $B=c^{\prime} R$ with $c^{\prime} \in \Sigma$ (see Lemma 1.4(5)). Then $r c^{\prime} R \leq c R$, and hence $r c^{\prime}=c r^{\prime}$ for $r^{\prime} \in R$.

Let $R_{*}=R_{\Sigma}$, the localization of $R$ with respect to $\Sigma$, and identify $R$ with its image in $R_{*}$. One can easily check that $S_{*}$ is an essential right ideal in $R_{*}$, and is semisimple as a right $R_{*}$-module. Thus $S_{*}$ is the right socle of $R_{*}$. We prove that $S_{*}$ is a maximal right ideal. Clearly $S_{*} \neq R_{*}$. If $S_{*}<M$ for some right ideal $M$ of $R_{*}$, then $M=D R_{*}$ for a right ideal $D$ of $R$ with $S<D$. Hence $D=d R$ with $d \in \Sigma$, and $M=d R R_{*}=d R_{*}=R_{*}$.

Next we prove that $R_{*}$ is right pseudo-semisimple. Let $A$ be a right ideal of $R$. Then $A \leq S$ or $A=a R$ with $a^{0}=0$. Thus $A R_{*} \leq S_{*}$ or $A R_{*}=a R R_{*}=a R_{*} \cong R_{*}$.

Let $x \in Z\left(R_{*}\right)$. Then $x S_{*}=0$, and hence $x S=0$. Since $x=r c^{-1}$ for some $c \in \Sigma, r S=x c S=x S=0$. Thus $r \in Z=0$, and hence $x=0$.

Remark. We note that in Proposition $2.6, \Sigma$ is actually the largest right Öre set of $R$, and hence $R_{*}$ is the maximal right classical ring of fractions of $R$.

\section{Subrings of full linear rings}

If $R$ is a ring with $Z=0$, then the maximal right quotient ring of $R$ is a regular right self-injective ring having $R$ as a subring. Moreover if $S$ is 
essential in $R$, then $Q=$ End $S_{R}$, and is therefore a product of full linear rings (compare [5, Chapter 12]); it is just one full linear ring if and only if $S$ is homogeneous.

In this section we discuss the existence of pseudo-semisimple rings which are subrings of full linear rings; in view of Lemma 1.5(2), non-trivial examples can only occur with linear rings of infinite dimensional vector spaces.

Throughout this section, $Q$ will stand for the endomorphism ring of a vector space $V$ of infinite dimension over a division ring $D$. We shall call an element $t \in Q$ a shift endomorphism if it is an isomorphism onto a subspace of codimension one. For such $t$ we choose a complement $U$ of $t V$, so that $V=t V \oplus U$ and $\operatorname{dim} U=1$. We define $t^{*}=t^{-1}$ on $t V$ and $t^{*}=0$ on $U$. Let $e=1-t t^{*}$. Then $e$ is the projection onto $U$ along $t V$, and hence is of rank one. A subring $R$ of $Q$ is said to contain a shift, if $t, t^{*} \in R$ for some shift $t$; it is clear that $e \in R$ and $(1-e) R \cong R$ (also $R(1-e) \cong R)$.

LEMMA 3.1. Let $L$ be a non-zero left ideal of $Q$ consisting of linear transformations of finite rank, and let $T$ be a subring of $Q$ having $L$ as a two sided ideal. If $T$ contains a shift and $T / L$ is a division ring, then $T$ is a right pseudo-semisimple ring with $S(T)=L$ and $Z(T)=0 ;$ moreover $T$ is regular if and only if $\cap\{\operatorname{ker} x: x \in L\}=0$.

Proof. Let $f$ be an indecomposable idempotent in $L$. Since $L$ is a left ideal in $Q, f$ stays indecomposable in $Q$, and therefore $f Q$ is a minimal right ideal. Let $f t \neq 0$ for some $t \in T$. Then there exists $q \in Q$ with $f t q=f$. Consequently $f t q f=f$ and $q f \in L \leq T$. This proves that $f T$ is a minimal right ideal in $T$.

Given $x \in L$, there exists $p \in Q$ such that $x p x=x$. Then $g=p x$ is an idempotent in $L$, and $x T=x g T \cong g T$. One may write $g=g_{1}+\cdots+g_{n}$ where the $g_{i} \in Q$ are orthogonal idempotents of rank one. However $g_{i}=$ $g_{i} g \in L$, and it follows by the preceding argument that $g_{i} T$ is a minimal right ideal. Therefore $x T \cong g T$ is semisimple. Hence $L$ is contained in the right socle of $T$. Since $T$ contains a shift, $T$ is not semisimple. Then $T / L$ is a division ring implies that $L$ is the right socle of $T$.

Our argument also shows that any minimal right ideal in $L$ is generated by a rank one idempotent. Since rank one idempotents are isomorphic in $Q$, they are also isomorphic in $T$ (again since $L$ is a left ideal in $Q$ ). Thus $L$ is homogeneous. Let $t$ be the given shift in $T$. Then it is clear that the rank one idempotent $e=1-t t^{*}$ is in $L$, and hence all rank one idempotents in $L$ are isomorphic to $e$. Then $T$ is right pseudo-semisimple by Theorem 2.2 . 
Given $0 \neq y \in Q$, then $0 \neq y Q e \leq L \leq T$ and $Q e \leq L \leq T$. Hence $T_{T}$ is essential in $Q_{T}$, and therefore $Z(T)=0$. (This also proves that $Q$ is the maximal quotient ring of $T$.)

Now we prove the last statement of the theorem. In view of Lemma 1.5(2) and Lemma 2.1(6), $T$ is regular if and only if $L^{0}=0$. Let $W=$ $\bigcap\{\operatorname{ker} x: x \in L\}$. If $W \neq 0$, then there exists $q \in Q$ such that $0 \neq q e V \leq$ $W$ (since $Q e V=V$ ), and therefore $0 \neq q e \in L^{0}$; thus $L^{0} \neq 0$. Conversely, assume that $W=0$ and let $r \in L^{0}$. Then $r V \leq \operatorname{ker} x$ for every $x \in L$, and therefore $r=0$. This proves that $L^{0}=0$ holds if and only if $W=0$.

At this point it is convenient to discuss some examples. We start with [2, Example 4.26], which is originally due to G. M. Bergman, and represents a regular, but not unit-regular ring, in which perspectivity is transitive. This example was suggested to us by $\mathbf{K}$. $\mathbf{R}$. Goodearl through a communication by K. M. Rangaswami. Similar examples can be obtained from the more general construction to be discussed in Proposition 3.5.

EXAMPLE 3.2. (A regular right and left pseudo-semisimple ring which is not semisimple.) Let $V=F[[t]]$, the power series ring over a field $F$ considered as an $F$-space, $Q=$ End $V_{F}$, and $F((t))$ the Laurent series ring, that is, the quotient field of $F[[t]]$. Let

$$
\begin{aligned}
& L=\left\{x \in Q: \exists n \in \mathbb{N}\left(x t^{n} V=0\right)\right\} \\
& T=\left\{x \in Q: \exists n \in \mathbb{N}, a \in F((t))\left((x-a) t^{n} V=0\right)\right\} .
\end{aligned}
$$

It is obvious that $t$ is a shift and $t, t^{*} \in T$. One can verify that $L$ is a left ideal of $Q$ consisting of linear transformations of finite rank, $T$ is a subring of $Q$ having $L$ as a two sided ideal and $T / L \cong F((t))$. Moreover $\bigcap\{\operatorname{ker} x: x \in L\}=\bigcap_{n \in \mathbb{N}} t^{n} V=0$. Thus $T$ is right pseudo-semisimple and regular by Lemma 3.1. According to Corollary 2.3, $T$ is also left pseudosemisimple.

EXAMPLE 3.3. (A non-singular right pseudo-semisimple ring which is not left pseudo-semisimple.) Modifying the above example by taking $V=$ $F[[t]] \oplus F((t))$, one obtains a right pseudo-semisimple ring $T$ with $Z(T)=$ 0 . However

$$
J(T)=\bigcap\{\operatorname{ker} x: x \in L\}=F((t)) .
$$

Thus $T$ is not left pseudo-semisimple in view of Corollary 2.3.

A right pseudo-semisimple ring $R$ in which $Z \neq S$ satisfies $0 \leq Z \leq$ $J<S$. Examples 3.2 and 3.3 correspond to the cases $0=Z=J$ and $0=Z<J$, respectively. Examples of the other two cases can be obtained using split extensions. 
Let $A$ be any right pseudo-semisimple ring with $Z(A)=0$ and $A / S(A)$ a division ring. Let $R=A \rtimes A / S(A)$. Then $R$ has right singular ideal $0 \rtimes A / S(A)$, right socle $S(A) \rtimes A / S(A)$ and Jacobson radical $J(A) \rtimes A / S(A)$; and $R$ is right pseudo-semisimple by Proposition 2.4 .

For the case $0<Z=J$ (respectively $0<Z<J$ ), take $R=T \rtimes T / L$ where $T$ is the ring of Example 3.2 (respectively 3.3).

LeMMA 3.4. Let $t \in Q=$ End $V_{D}$ be a shift. If $q$ is a non-zero polynomial over the centre of $D$, then $q(t)$ has infinite rank.

Proof. Let $q=a_{m} X^{m}+\cdots+a_{n} X^{n}$ where $m \leq n$ and $a_{m} \neq 0$. Without loss of generality we may assume that $a_{m}=1$. Let $K=\operatorname{ker} q(t)$. Then clearly $t^{i} K \leq K$ for every $i \in \mathbb{N}$. Now

$$
0=t^{* m} q(t) K=\left(1+a_{m+1} t+\cdots+a_{n} t^{n-m}\right) K,
$$

and so $K \leq t K$. Thus $K=t^{i} K \leq t^{i} V$ for every $i \in \mathbb{N}$. Writing $V=t V \oplus U$, we get

$$
V=t^{i} V \oplus t^{i-1} U \oplus \cdots \oplus t U \oplus U
$$

Therefore $K \cap \bigoplus_{i=0}^{\infty} t^{i} U=0$, and hence $K$ has infinite codimension. Thus $q(t)$ is of infinite rank.

Let $A$ denote the prime subring of $D$, that is, the subring of $D$ generated by the identity element. We shall say that the pair $(t, L)$ is permissible if $t$ is a shift endomorphism and $L$ a non-zero left ideal of $Q$ consisting of linear transformations of finite rank such that:

$\left(\mathrm{P}_{1}\right) \forall 0 \neq q \in A[X] \forall x \in Q(x \in L \Leftrightarrow x q(t) \in L)$;

$\left(\mathrm{P}_{2}\right) \forall 0 \neq q \in A[X] \exists y \in L(\operatorname{ker} q(t) \cap \operatorname{ker} y=0)$.

REMARK. One particular choice of $L$ is the ideal consisting of all linear transformations of finite rank. For this choice, a shift $t \in Q$ is such that $(t, L)$ is permissible if and only if $\operatorname{ker} q(t)$ has finite dimension and $\operatorname{Im} q(t)$ has finite codimension for all $0 \neq q \in A[X]$.

A shift $t$ satisfying the above requirements exists in every full linear ring $Q=$ End $V_{D}$. Indeed $A[X]$ is countable, and therefore the central localization $D\left[X, X^{-1}\right]_{*}$ at the non-zero elements of $A[X]$ is a countable dimensional $D$-space. Consequently $V \cong D[X] \oplus D\left[X, X^{-1}\right]_{*}^{(\operatorname{dim} V)}$. Define $t$ as componentwise multiplication by $X$. This yields a shift with $\operatorname{ker} q(t)=0$ for every $0 \neq q \in A[X]$. Moreover, if $q$ is of degree $n$, then by the Euclidean Algorithm

$$
D[X]=q(X) D[X] \oplus D \oplus D X \oplus \cdots \oplus D X^{n-1} .
$$


Clearly $q[X] D\left[X, X^{-1}\right]_{*}=D\left[X, X^{-1}\right]_{*}$. Therefore $\operatorname{Im} q(t)$ has finite codimension.

The following proposition ensures that subrings as described in Lemma 3.1 exist in every infinite-dimensional full linear ring.

Proposition 3.5. Let $(t, L)$ be a permissible pair. Then

$$
T=\{x \in Q: \exists p, 0 \neq q \in A[X](x q(t)-p(t) \in L)\}
$$

is a non-singular right pseudo-semisimple ring with $L$ as its right socle and $T / L \cong A(X)$, the quotient field of $A[X]$.

Proof. From Lemma 3.4 and condition $\left(P_{1}\right)$, a routine verification establishes that $T$ is a subring of $Q$, and $\varphi: x \rightarrow p / q$ is a well defined ring homomorphism of $T$ into $A(X)$. To show that $\varphi$ is surjective consider any $p / q \in A(X)$. By $\left(\mathrm{P}_{2}\right)$ we have $y \in L$ such that $\operatorname{ker} q(t) \cap \operatorname{ker} y=0$. Hence $q(t)_{\mid \text {ker } y}$ is one-to-one. Let $V=q(t) \operatorname{ker} y \oplus C$. Then the mapping $x$ given by

$$
x(q(t) v)=p(t) v, \quad v \in \operatorname{ker} y ;\left.x\right|_{C}=0
$$

is a well defined element in $Q$. Let $\alpha=x q(t)-p(t)$. Then $\left.\alpha\right|_{\text {ker } y}=0$. Write $V=\operatorname{ker} y \oplus W$. Since $y W \cong W$, there exists $\beta \in Q$ such that $\left.\beta y\right|_{W}=1$. It then follows that $\alpha=\alpha \beta y \in L$. This shows that $x \in T$ as well as $\varphi(x)=p / q$.

It is clear that $\operatorname{ker} \varphi=\{x \in Q: \exists 0 \neq q \in A[X](x q(t) \in L)\}$. Then by $\left(\mathrm{P}_{1}\right), L=\operatorname{ker} \varphi$. Hence $L$ is an ideal in $T$ and $T / L \cong A(X)$. Now $t 1-t=0$ and $t^{*} t-1=0$ imply that $t, t^{*} \in T$. The result now follows from Lemma 3.1.

THEOREM 3.6. $A$ ring $R$ is a non-singular right pseudo-semisimple ring with homogeneous maximal socle if and only if

(1) $R$ is a subring of a full linear ring $Q$,

(2) there exists a permissible pair $(t, L)$ in $Q$ with $L$ an ideal in $R$ and $R / L$ a division ring, and

(3) the ring $T$ corresponding to $(t, L)$, as in Proposition 3.5, is a subring of $R$.

Proof. The 'if' part follows from Lemma 3.1 as $t, t^{*} \in T \leq R$.

'Only if'. Since $R$ is right non-singular and $S$ is homogeneous, the maximal quotient ring $Q$ of $R$ is a full linear ring; $Q=$ End $V_{D}$. Also $Q \cong$ End $S_{R}$, and hence $S$ is a left ideal of $Q$ consisting of linear transformations of finite rank. By Theorem 2.2, $R$ has a shift for some indecomposable idempotent $e \in S$. As $e$ is a rank one projection, $t$ is a shift 
endomorphism in $Q$. We verify conditions $\left(\mathrm{P}_{1}\right)$ and $\left(\mathrm{P}_{2}\right)$ for the pair $(t, S)$.

Let $0 \neq q \in A[X]$. Then $q(t)$ is not of finite rank by Lemma 3.4 and hence not in $S$. It follows by Lemma $2.1((2)$ and (3)) that $R=f R \oplus(1-f) R$ such that $q(t) R=f R$ and $1-f \in S$. Let $f=q(t) r, r \in R$. Then $r q(t)$ is also an idempotent, and $r q(t) \notin S$; otherwise $q(t)=f q(t)=q(t) r q(t) \in S$, a contradiction.

For the non-trivial implication of $\left(\mathrm{P}_{1}\right)$, assume that $x q(t) \in S$ for some $x \in Q$. Then $x f=x q(t) r \in S$. Also $x(1-f) \in S$. Thus $x \in S$.

To prove $\left(\mathrm{P}_{2}\right)$, note that $1-r q(t) \in S$ by Lemma 2.1(3). Clearly ker $q(t) \cap$ $\operatorname{ker}(1-r q(t))=0$.

Now $\left(P_{1}\right)$ and $\left(P_{2}\right)$ being established, we may form the subring $T$ of $Q$ according to Proposition 3.5. Let $x \in T$. Then $x q(t)-p(t)=s \in S$, for some $p, 0 \neq q \in A[X]$. With $f$ and $r$ as before, we obtain

$$
x f=x q(t) r=(p(t)+s) r \in R,
$$

and $x(1-f) \in S \leq R$. Hence $x \in R$.

\section{Added in Proof}

Using Lemma 3.1, the referee suggested the following example of a regular pseudo-semisimple ring which is not semisimple (a similar example was suggested by Mark L. Teply). Let $Q$ be the ring of $\aleph_{0} \times \aleph_{0}$ column-finite matrices over a field $F$, let $L=$ Socle $Q$ (set of matrices with a finite number of non-zero rows), and let $M$ be the subset of $Q$ consisting of all matrices of the form.

$$
\left[\begin{array}{llllllll}
a_{0} & a_{1} & a_{2} & & & & \\
b_{1} & a_{0} & a_{1} & a_{2} & . & . & . \\
b_{2} & b_{1} & a_{0} & a_{1} & a_{2} & & \\
\cdot & b_{2} & b_{1} & a_{0} & & & \\
\cdot & & b_{2} & & &
\end{array}\right],
$$

where only a finite number of the $b_{i}$ are non-zero. Let $T=L+M$. It is clear that $T$ is a ring which contains the standard shift

$$
t=\left[\begin{array}{cccccc}
0 & & & & & \\
1 & & & & \\
& 1 & & & \\
& & 1 & & \\
& & & . & \\
& & & & . & \\
& & & & & .
\end{array}\right] \text {, }
$$


$L$ is a two sided ideal in $T$, and $T / L \cong F\left(\left(t^{*}\right)\right)$ where

$$
t^{*}=\left[\begin{array}{cccccc}
0 & 1 & & & & \\
& & 1 & & & \\
& & & 1 & & \\
& & & 1 & \\
& & & & & \ddots
\end{array}\right] \text {. }
$$

The authors are thankful to the referee for other comments and suggestions.

\section{References}

[1] J. S. Golan, Localizations of non-commutative rings, (Marcel Dekker, 1975).

[2] K. R. Goodearl, Von Neumann regular rings, (Pitman, 1979).

[3] Saad Mohamed and Bruno J. Müller, 'Pseudo-semisimple rings', Proc. Amer. Math. Soc. 85 (1982), 157-160.

[4] Saad Mohamed and Surjeet Singh, 'Weak $q$-rings with zero singular ideals', Proc. Amer. Math. Soc. 76 (1979), 25-30.

[5] Bo Stenström, Rings of quotients, (Springer Verlag, 1975).

Department of Mathematics

Kuwait University

P.O. Box 5969

Kuwait 13060
Department of Mathematics

McMaster University

Hamilton

Ontario L8S 4K1

Canada 